Exercices Exercices de rhétorique

de rhétorique $\quad 6 \mid 2016$

Sur l'épistolaire

\title{
La rhétoricité conditionnelle des lettres de Mme de Sévigné
}

\section{Cécile Lignereux}

\section{(2) OpenEdition \\ 1 Journals}

Édition électronique

URL : http://journals.openedition.org/rhetorique/428

DOI : 10.4000/rhetorique.428

ISSN : 2270-6909

Éditeur

UGA Éditions/Université Grenoble Alpes

Édition imprimée

ISBN : 978-2-84310-316-2

\section{Référence électronique}

Cécile Lignereux, «La rhétoricité conditionnelle des lettres de Mme de Sévigné », Exercices de rhétorique [En ligne], 6 | 2016, mis en ligne le 10 février 2016, consulté le 12 septembre 2020. URL http://journals.openedition.org/rhetorique/428 ; DOI : https://doi.org/10.4000/rhetorique.428

Ce document a été généré automatiquement le 12 septembre 2020.

\section{(c) (1) () (2)}

Les contenus de la revue Exercices de rhétorique sont mis à disposition selon les termes de la Licence Creative Commons Attribution - Pas d'Utilisation Commerciale - Partage dans les Mêmes Conditions 4.0 International. 


\title{
La rhétoricité conditionnelle des lettres de Mme de Sévigné
}

\author{
Cécile Lignereux
}

1 Dans le sillage de la distinction fondatrice de Gérard Genette, il est possible de distinguer "deux régimes» de rhétoricité : «le constitutif, garanti par un complexe d'intentions, de conventions génériques, de traditions culturelles de toutes sortes », et «le conditionnel, qui relève d'une appréciation [...] subjective et toujours révocable ${ }^{1}$ ». Assurément, ce n'est que de manière conditionnelle, c'est-à-dire en vertu d'un jugement se fondant sur des «critères » propres à caractériser différents « modes $^{2}$ » de rhétoricité, que les lettres de Mme de Sévigné peuvent être perçues, à divers titres, comme rhétoriques - ce qui engage toute une réflexion sur la légitimité du mécanisme qui conduit de la recherche empirique de traces, d'indices et de marqueurs au diagnostic de rhétoricité.

2 Postuler la rhétoricité conditionnelle des lettres de Mme de Sévigné (quels qu'en soient les degrés et les échelles) : tel est le parti-pris des articles rassemblés dans ce numéro. Résolument situés du côté de l'expérience lectoriale, tous présupposent que le texte épistolaire est «connaissable», c'est-à-dire qu'il contient des "moyens» et des « techniques » qui peuvent «faire sens ${ }^{3}$ ». Centrées sur les effets produits à réception, c'est-à-dire "ne prétend[ant] pas décrire le "contenu" des lectures possibles, mais les procédures textuelles qui rendent ces lectures possibles ${ }^{4}$ ", ces contributions mettent en œuvre un art de lire qui ne consiste ni à « chercher naïvement la "bonne" lecture ", ni à "valoriser systématiquement l'indécidable», mais, plus pragmatiquement, à « examiner, analyser, décrire les lieux où le texte permet la dérive, les lieux où il contraint, les lectures qu'il propose, celles qu'il refuse, celles qu'il laisse indéterminées ou incertaines ${ }^{5} »$. Aussi variés que soient les angles d'attaque de chacune de ces contributions, toutes illustrent ainsi les retombées heuristiques d'un savoir-lire visant à repérer les dispositifs qui, à la lecture, orientent vers un certain sens.

Pour que puissent advenir des investigations textuelles pariant sur notre capacité à percevoir les procédures qui président aux effets du texte, encore fallait-il que soient mis à distance toutes sortes de réflexes herméneutiques qui ne peuvent que faire écran 
aux modalités formelles des lettres de Mme de Sévigné. Or voici que dans la vaste économie des interprétations dont la Correspondance a fait l'objet ${ }^{6}$ (chacune étant forcément aussi stimulante que limitée), toutes les conditions semblent désormais réunies pour que puissent être mises au jour les procédures formelles constitutives de la lisibilité épistolaire. S'il y a aujourd'hui place pour d'autres protocoles de lecture que la paraphrase psychologisante dans laquelle s'est longtemps cantonnée la critique sévignéenne (protocoles qui tendent heureusement à se répandre dans le paysage critique contemporain ${ }^{7}$ ), c'est en raison de la convergence de trois acquis méthodologiques qui ont en commun non seulement de permettre mais encore de favoriser l'examen raisonné des caractéristiques formelles de la Correspondance.

D'une part, il fallait opérer une conversion du regard afin de dépasser les grilles de lecture psycho-biographiques devenues hégémoniques et ainsi pouvoir saisir la Correspondance non pas à travers les intentions à jamais inaccessibles de la marquise mais à partir des seuls énoncés textuels. D'autre part, il était nécessaire de rompre avec l'idée selon laquelle les lettres de Mme de Sévigné, appréhendées à l'aune d'idéaux socio-culturels indissociables de jugements de valeur, échapperaient à toute mise en forme - ce qui implique de résister à des fantasmes critiques propres à oblitérer la dimension textuelle de la Correspondance. Enfin, pour décrire des formes textuelles, ce qui implique de repérer des indices embrayeurs d'effets, il était indispensable de comprendre que tout parcours dans un univers textuel passant par la mobilisation de grilles de lecture, il est utile de disposer de modèles d'organisation extérieurs pour expérimenter, dans la perspective d'une rhétorique de la lecture, les dispositifs possibles du texte. Tels sont les trois principes méthodologiques que nous explorerons successivement au cours de cette présentation.

\section{Place aux approches formelles}

5 Sans doute plus que tout autre texte, la Correspondance de Mme de Sévigné (dont la place éminente dans le Panthéon littéraire français est le fruit d'une canonisation pleine de malentendus, où les présupposés de tous ordres ${ }^{8}$ tiennent autant de place que les aléas rocambolesques d'une correspondance authentique ${ }^{9}$ ) s'avère "marqué[e] d'une essentielle précarité ${ }^{10} »-$ ce qui ne saurait qu'encourager l'exploration de sens nouveaux. Que la Correspondance, en raison de son statut aussi instable et fuyant que les «images ${ }^{11}$ » de la marquise, soit vouée à susciter des modes de lecture et d'interprétation aussi renouvelés que contrastés : telle est finalement la leçon que l'on retiendra du fameux débat qui opposa Bernard Bray et Roger Duchêne ${ }^{12}$. On se souvient que tandis que Bernard Bray mettait en avant l'avènement d'un style qui déploierait son propre système esthétique, derrière lequel se lirait en creux des motivations artistiques propres aux seuls écrivains, Roger Duchêne insistait sur la spontanéité d'une écriture qui, loin de toute préoccupation d'ordre esthétique, répondrait seulement à des exigences sentimentales.

6 Certes, dans un premier temps, ce débat n'a pas manqué de générer bon nombre d'ornières qui ont longtemps hanté la critique sévignéenne: en gravitant autour de notions aussi problématiques que celles de la conscience littéraire de la marquise, de sa sincérité, de sa vocation d'écrivain ou de la place du vécu par rapport à l'art, une telle controverse condamnait à formuler des alternatives aussi caricaturales qu'aporétiques (travail stylistique conscient ou spontanéité ? vocation littéraire ou amour maternel? 
recherche esthétique ou pure affectivité ?). Pourtant, près d'un demi-siècle plus tard, à l'heure où chacun s'accorde à reconnaître qu'il vaut mieux neutraliser les considérations d'ordre psychologique autour des intentions de l'épistolière, on reconnaîtra que cette polémique a l'avantage d'avoir révélé combien la Correspondance, en raison non seulement des ambiguïtés inhérentes à tout genre factuel mais aussi de ses fragilités philologiques insurmontables ${ }^{13}$, a pour effet de susciter des attitudes lectoriales aussi variées qu'irréductibles. Autrement dit, une fois fait le deuil de questionnements voués à rester irrésolus (on conviendra aisément que la question des intentions de Mme de Sévigné ne trouvera jamais de réponse satisfaisante), il devient possible d'expérimenter des systèmes de lecture qui, en se situant du côté non plus de l'écriture mais de la lecture, non plus de la production mais de la réception, non plus des intentions mais des effets, prennent pour seul point de départ les procédés textuels.

Prendre conscience des impasses auxquelles condamnent les spéculations sur les intentions de l'épistolière constitue la condition de possibilité sine qua non des approches formelles de la Correspondance. Néanmoins, ce n'est là qu'une première étape : pour parvenir à prendre en charge la Correspondance comme texte, c'est-à-dire comme ensemble organisé, encore faut-il se débarrasser de l'idée selon laquelle les lettres de Mme de Sévigné seraient informes. En effet, la méfiance qu'inspirent les considérations psycho-biographiques ne suffit pas à nous préserver d'impressions de lecture liées non pas à de rigoureux repérages textuels mais à des hypostases critiques d'obédience vaguement sociologique.

\section{Non, les lettres de Mme de Sévigné ne sont pas informes}

8 Il y a quelques années, Christine Noille posait une question aussi provocante que salutaire : «les lettres de Sévigné sont-elles informes ?14 ». Au-delà d'une formulation cocasse ayant le mérite de questionner de front la pertinence d'habitudes herméneutiques lourdes de présupposés mal élucidés, un tel titre constitue une authentique mesure prophylactique destinée à contrer la propagation de l'idée selon laquelle les lettres de Mme de Sévigné seraient exemptes de toute opération de mise en forme, de disposition, d'agencement - une idée qui, dans l'histoire de la critique sévignéenne, pèse aussi lourd que celle d'une écriture n'engageant pas d'autres ressources que celles du cœur.

9 Il faut dire que les nombreuses déclarations de Mme de Sévigné soulignant avec panache le caractère primesautier de son écriture n'ont pas manqué de décourager les tentatives d'analyse formelle. Ces déclarations, qui revendiquent, non sans coquetterie, un mode d'écriture délivré de toute inhibition, sont bien connues - qu'elles insistent sur la désinvolture d'un style déconcertant de souplesse et de variété («mon style est si négligé qu'il faut avoir un esprit naturel et du monde pour s'en pouvoir accommoder ", 23 décembre 1671: I, 398 ${ }^{15}$ ); sur l'accumulation aléatoire et hétéroclite de sujets juxtaposés sur le mode d'infinis «fagotages» (4 mars 1671: I, 449), « rapsodies » (10 juin 1671: I, 271) ou «fatras de bagatelles» (15 juin 1680: II, 973); sur le flux irrépressible de missives disproportionnées ("J'écris sans mesure », 9 mars 1672: I, 456); sur la teneur confidentielle de la " causerie » (14 décembre 1689: III, 779) et des « verbiages » (5 janvier 1676 : II, 214); sur la rapidité d'une rédaction au fil de la plume (« Est-il possible, ma chère fille, que j'écrive bien ? Cela va si vite !», 20 décembre 1688 : 
III, 437); sur la progression erratique d'une rédaction sans relecture régulière ("Quelquefois même il arrive une singulière chose, c'est qu'oubliant ce que je vous ai mandé au commencement de ma lettre, j’y reviens encore à la fin », 11 mars 1672: I, $457)$; sur l'absence de maîtrise et l'abandon au plaisir d'écrire («En vérité, il faut un peu, entre bons amis, laisser trotter les plumes comme elles veulent; la mienne a toujours la bride sur le cou. », 24 novembre 1675 : II, 170).

Parce que Mme de Sévigné ne cesse de mettre en exergue le caractère impulsif et désordonné de ses lettres, il est tenu pour une vérité que le texte de la Correspondance échappe à tout principe formel — ce qui laisse songeur quant à l'alignement irréfléchi de nos pratiques de lecture sur notre prétendu savoir concernant le processus d'écriture épistolaire. Pour le dire brutalement, mieux vaut ne pas accorder trop de crédit aux séduisantes déclarations d'une épistolière qui se targue d'écrire «naturellement, et sans [s'en] faire une affaire» (6 octobre 1679: II, 698). De fait, lorsque l'on décide de ne pas être dupe de protestations épistolaires dont la plaisante désinvolture est largement topique à l'époque de la marquise, force est de constater qu'il est aisé de repérer des indices irréfutables d'un constant souci accordé à la gestion de la lisibilité, que ceux-ci soient perceptibles au niveau local d'unités textuelles autonomes analysables en termes de séquences (séquences d'anecdotes, de réflexions morales ou de nouvelles ${ }^{16}$ ) ou au niveau global du tissu épistolaire (qu'il s'agisse de dispositifs de cohésion, de structuration par le rythme ou de délimitation $\left.{ }^{17}\right)$. Le fait que les lettres de Mme de Sévigné soient loin d'être dépourvues d'opérateurs de structuration incite ainsi à la plus grande prudence quant à leur caractère supposé spontané et imprévisible.

11 Or pour pouvoir repérer dans le texte de la Correspondance des dispositifs rhétoriques embrayeurs de lisibilité, il ne suffit pas de dépasser la tentation de croire sur parole Mme de Sévigné - même si c'est un préalable nécessaire. Encore faut-il congédier les illusions d'inspiration biographique, les mythes esthétiques et les frilosités épistémologiques - obstacles qu'il s'agit d'examiner l'un après l'autre. Ce n'est en effet qu'au prix d'une remise en question d'un certain nombre de fausses évidences que l'on peut systématiser le geste qui consiste à relever les indices textuels embrayeurs de formalisation, à quelque niveau que ce soit.

\subsection{Au-delà des illusions d'inspiration biographique}

12 Le désintérêt pour les techniques de formalisation épistolaire semble se nourrir de tenaces idées reçues concernant l'art d'écrire de Mme de Sévigné, ou plus précisément le genre que celle-ci pratique (la lettre familière) et le sexe de l'épistolière (qui a « la chance d'être femme $\left.{ }^{18} »\right)$. Or pour saisir les différentes dynamiques formelles à l'œuvre dans la Correspondance, mieux vaut prendre comme seul point de départ les procédés textuels plutôt que de spéculer sur les compétences, les goûts et les aspirations de la marquise, en alléguant par exemple le fait qu'elle ne connaît ni les recueils de lettres ni les manuels épistolographiques des générations précédentes ${ }^{19}$; qu'elle ne s'intéresse ni aux chefs-d'œuvre épistolaires alors en vogue ni aux correspondances érudites qu'entretiennent ses proches (Chapelain, Ménage) ${ }^{20}$, Voiture étant «le seul épistolier qui fasse partie de sa culture ${ }^{21} »$; qu'elle ne saurait goûter que la spontanéité et le naturel, conformément aux idéaux de la mondanité galante ${ }^{22}$; ou encore qu'elle peut 
laisser libre cours à une inventivité non bridée par les normes enseignées aux hommes dans les collèges ${ }^{23}$.

Certes, l'écriture sévignéenne ne reproduit pas servilement les agencements institués (qu'il s'agisse des canevas standards répertoriés par les manuels d'art épistolaire ou des stratégies discursives recensées par les traités de rhétorique). Pourtant, considérer que quelques supputations autour de la personnalité de l'épistolière, de ses ressources et de ses motivations, suffisent à rendre non pertinente toute investigation formelle expose à de redoutables erreurs de perspective sur le fonctionnement et l'efficacité du texte de la Correspondance. Constater que Mme de Sévigné ne convoque pas des protocoles dont le conformisme empesé n'est pas adapté à son projet communicationnel n'autorise pas pour autant à conclure à une absence de travail formel. Pour le dire autrement, ce n'est pas parce que les lettres de Mme de Sévigné n'actualisent pas de manière ostensiblement conventionnelle les stéréotypes consignés par les manuels qu'elles sont pour autant exemptes d'instruments de lisibilité.

Lorsque l'on sort d'une vision caricaturale des formes épistolaires, c'est-à-dire lorsque l'on comprend que l'écriture épistolaire se situe à l'opposé aussi bien d'une soumission conformiste aux usages scrupuleusement réglés de la bienséance que d'une singularisation de soi à la fois périlleuse et inconcevable, on s'aperçoit que de nombreux éléments viennent accréditer l'idée d'un travail de mise en forme - nous nous contenterons d'en mentionner deux. D'une part, les nombreuses réflexions métadiscursives qui jalonnent les lettres de Mme de Sévigné attestent une constante attention aux formes d'écriture - que ces réflexions soient interprétées comme le reflet de préférences esthétiques ${ }^{24}$ ou envisagées comme autant de négociations visant à assurer l'harmonie communicationnelle entre les correspondants ${ }^{25}$. D'autre part, les lettres de Mme de Sévigné mobilisent de nombreuses pratiques langagières culturellement normalisées, socialement ritualisées et linguistiquement codifiées. Pour peu que l'on maîtrise les préconstruits rhétoriques sous-jacents, il est possible de repérer et d'identifier dans la Correspondance toutes sortes de rituels socio-discursifs qui, grâce à de nombreux procédés d'assouplissement des conventions (phénomènes d'écarts, d'hybridations ou de variations), se trouvent acclimatés à la lettre familière - citons par exemple la recommandation ${ }^{26}$, l'offre de service, la remontrance ou encore l'exhortation ${ }^{27}$. Une fois mis à distance les préjugés d'inspiration biographique sur la familiarité épistolaire à l'âge classique, il devient ainsi possible de déceler les indices textuels d'une authentique activité formelle.

\subsection{Au-delà des mythes esthétiques}

15 Vaincre la résistance aux approches formelles des lettres de Mme de Sévigné implique également de surmonter l'obstacle qui consiste à percevoir la Correspondance non pas à partir d'effets objectivement descriptibles et assignables à des lieux précis du texte, mais à travers le filtre de mythes esthétiques qui ne font que renchérir sur des options idéologiques. Certes, reconnaître au style de Mme de Sévigné, comme cela a été si souvent fait, une aisance, une délicatesse ou encore une facilité caractéristiques de l'art de plaire galant n'est pas entièrement illusoire. Pourtant, force est de constater que les appréciations du style de Mme de Sévigné qui se fondent sur des idéaux socio-culturels indissociables de jugements de valeur (ceux du classicisme) ont surtout pour effet de détourner l'attention des procédés textuels - les catégories critiques laudatives 
systématiquement appliquées au style sévignéen depuis trois siècles n'étant que prétexte à de larges vues surplombantes qui ne s'arrêtent pas au détail du texte.

C'est surtout le mythe fédérateur du naturel qui a constitué un obstacle majeur aux investigations formelles. Le fameux naturel sévignéen n'a pas manqué de faire couler beaucoup d'encre : trois types d'analyse, tributaires de postulats hétérogènes, ont tenté d'en définir les fondements. Le naturel est ainsi rapporté tantôt au milieu social de la marquise (il résulterait $\mathrm{du}$ modèle de la conversation mondaine, où règnent les exigences de naïveté et de négligence ${ }^{28}$ ), tantôt au genre de l'épistolière (il découlerait de qualités spécifiquement féminines ${ }^{29}$ ), tantôt à l'authenticité des sentiments maternels (il serait la simple traduction d'une "réalité vécue ${ }^{30}$ )). Ce rapide survol des principes explicatifs couramment allégués pour justifier l'impression de naturel suffit à faire mesurer à quel point leur visée aussi totalisante qu'idéologiquement marquée ne pouvait que faire écran à la dimension textuelle des lettres de Mme de Sévigné.

Il peut bien y avoir divergence sur les origines attribuées au naturel sévignéen, il n'en reste pas moins que celui-ci fait généralement l'objet d'un consensus où prévaut un imaginaire de l'affranchissement et de l'émancipation. Or, pour attractif que soit cet imaginaire prompt à tomber dans le piège de la téléologie, il n'en est pas moins dangereusement anachronique - car à l'époque de Mme de Sévigné, «la liberté épistolaire n'est pas licence, mais récompense de la parfaite maîtrise d'une culture, et des possibilités du langage ${ }^{31} »$. C'est ce que signale avec force l'article de Bruno Méniel qui, en tête des contributions, rappelle que "le naturel, au XVII siècle, n'est pas le résultat de la spontanéité, de l'abandon, d'une facilité immédiate, mais procède d'un travail sur le langage, d'un art d'écrire. C'est l'effet de la culture la plus raffinée.» D'ailleurs, loin d'entraver la communication entre les correspondants, les conventions formelles (aussi allégées, croisées ou réinterprétées soient-elles) permettent au contraire, en organisant l'échange selon des principes partagés, de le sécuriser et de le fluidifier, les épistoliers de l'âge classique étant convaincus que "le laisser-aller ne conduirait qu'à l'informe " et "serait un obstacle à l'échange social», dont le déroulement harmonieux nécessite "un socle de conventions». Ainsi le naturel sévignéen, qui s'enracine dans un "refus de l'affectation et de l'artifice», doit-il être appréhendé comme résultant $d$ '« une adresse et une ingéniosité qui doivent rester invisibles ».

Le fait que Mme de Sévigné, congédiant les artifices usés et les banalités d'usage, ne mette pas systématiquement en œuvre les normes usuelles du bien-dire épistolaire de manière scolaire et voyante, n'autorise donc pas à conclure que la Correspondance ne mérite pas de faire l'objet d'enquêtes formelles. Une fois encore, il s'avère très imprudent de se fier aux séduisantes revendications par l'épistolière d'un «style naturel et dérangé » (3 février 1672: I, 428). Certes, Mme de Sévigné expérimente une "éloquence " faite de discrétion, de subtilité et de sobriété, à l'opposé de dispositifs aussi grandiloquents que convenus ${ }^{32}$ - on notera au passage que les nuances dans les degrés d'éloquence sont d'autant plus riches qu'elles peuvent considérablement varier non seulement d'une partie de la lettre à une autre mais encore au sein d'une même partie. Pourtant, à une époque où l'idéal de naturel, qui procède non pas d'un refus des normes et des usages établis, mais de leur appropriation, ne se confond pas avec un style original, il ne faut pas sous-estimer la prégnance de patrons discursifs dont les stéréotypes alimentent toutes sortes de variations. Pour peu que l'on fasse le pari d'une mobilisation d'usages langagiers conventionnels, il est possible de repérer dans le tissu 
de la Correspondance, à différentes échelles du texte, toutes sortes de matrices formelles (routines socio-linguistiques ${ }^{33}$, structures argumentatives ${ }^{34}$, figures rhétoriques ${ }^{35}$, moules syntaxiques ${ }^{36}$, procédés lexicaux ${ }^{37}$ ) qui, en attestant la reconfiguration et le réinvestissement de sédimentations discursives, viennent tordre le cou à l'idée d'un naturel pensé en termes de libération et de singularité. Tout en s'interdisant de spéculer sur les desseins de Mme de Sévigné, et en prenant comme seuls objets d'investigation les énoncés textuels, il ne semble donc pas déraisonnable d'enquêter sur la présence de formes signifiantes, en dépit de l'impression persistante de naturel.

\subsection{Au-delà des frilosités épistémologiques}

Thôt que l'on projette d'entreprendre une enquête sur la rhétoricité des lettres de Mme de Sévigné surgissent de lancinantes questions sur la légitimité d'une telle démarche. Avons-nous le droit de partir à la recherche configurations formelles? Est-il pertinent de procéder au relevé de procédés et de techniques? Est-ce un contresens que d'appréhender le texte de la Correspondance autrement qu'en le présupposant dépourvu d'agencements et de structures ? Si un tel excès de scrupule peut prêter à sourire (rares sont finalement les corpus à susciter autant de mauvaise conscience de la part de critiques ne faisant somme toute que reproduire les gestes habituels de l'explication de texte), il n'en révèle pas moins à quel point les systèmes de lecture de la Correspondance se trouvent spontanément indexés sur des représentations biographiques. La question de savoir s'il est légitime d'arpenter le terrain textuel de la Correspondance sous l'angle formel a donné lieu à deux prises de position antagonistes sur les ressources et les compétences de Mme de Sévigné en matière de rhétorique ${ }^{38}$.

D'un côté, il y a ceux qui pensent qu'il est incongru de partir à la recherche de dispositifs rhétoriques dans les lettres de Mme de Sévigné. Leur méfiance voire leur mépris à l'égard des techniques de mise en forme s'abreuve à trois considérations principales. D'abord, la situation d'énonciation propre à la lettre familière devrait bloquer nos réflexes formalistes, puisque «Mme de Sévigné écrit des lettres : elle ne pratique pas un genre littéraire ${ }^{39} "$, c'est-à-dire qu'elle n'écrit pas en auteur épistolaire mais seulement en épistolière ${ }^{40}$. Ensuite, le "ton» des lettres à Mme de Grignan, caractérisé par sa "vérité " et son "naturel», invaliderait l'hypothèse de préoccupations formelles : bien que Mme de Sévigné ait certes « eu recours aux façons d'exprimer l'amour habituelles à son temps" (communes aux lettres fictives et aux correspondances authentiques), « elle ne [les] a pas cherch[ées] dans ses livres, à peine dans sa mémoire ; elle [les] trouve dans son expérience vécue ${ }^{41} »$. Enfin, la formation et la culture de la marquise hypothèqueraient tout repérage de procédés rhétoriques, dans la mesure où «en ce siècle où la rhétorique tient une place si importante, la meilleure moitié du public, tout le beau sexe, l'ignore ou ne la connaît que superficiellement »: si Mme de Sévigné écrit aussi librement, c'est vraisemblablement moins parce qu'elle refuse les règles et les conventions que parce qu'elle les ignore, sa « négligence » n'allant pas « au-delà de la rhétorique », mais restant « en deçà ${ }^{42}$ ».

De l'autre côté, il y a ceux qui se donnent pour tâche de recenser les indices textuels de rhétoricité dans la Correspondance. Deux éléments allant dans le sens d'un travail proprement rhétorique sont à verser au dossier. D'une part, l'épistolière se soucie constamment de la réception de ses lettres, multipliant les procédés destinés à capter l'attention du destinataire à faciliter sa compréhension : "Mme de Sévigné ne néglige 
ni l'art de l'exorde, ni les ressources de la graphie, ni la description de la façon dont la lettre est matériellement composée, pour négocier au mieux la lisibilité de ses écrits ${ }^{43}$. " D'autre part, l'épistolière, lorsqu'elle commente des textes (aussi bien ceux qu'elle lit ou entend que ses propres lettres), fait grand usage des outils techniques de la rhétorique et de la poétique : elle manifeste non seulement « un art de remarquer et d'identifier formellement des séquences, ainsi qu'une sensibilité au ductus, à la conduite, au mouvement du discours » mais encore « un art de modéliser les textes lus selon des canevas-type ${ }^{44} »$.

Il est certes toujours possible de dépasser l'antithèse et de rendre l'alternative non exclusive en émettant sans trop de risque l'hypothèse relativement consensuelle d'un habitus propre au milieu social privilégié de la marquise : à l'opposé aussi bien d'un désintérêt pour la rhétorique que d'une mise en œuvre appliquée, c'est probablement par une forme d'imprégnation caractéristique de la culture aristocratique que Mme de Sévigné exploite les compétences diffusées par des traités et des manuels qu'elle n'a pas besoin de lire ${ }^{45}$. Pourtant, à tout bien considérer, la question de savoir si Mme de Sévigné connaissait la rhétorique et si elle la mobilisait sciemment dans la rédaction de ses lettres pose sans doute plus de problèmes qu'elle n'en résout. En enfermant dans des supputations biographiques autour du degré de planification et d'artificialité de l'écriture épistolaire, elle fait inévitablement ressurgir les apories concernant les intentions de la marquise. Dès lors, au lieu de s'ingénier à départager ce que Mme de Sévigné doit aux théoriciens, à ses fréquentations mondaines, à sa sensibilité esthétique ou à son affectivité, il est sans doute de bonne méthode de prendre le contre-pied de démarches participant d'une idéologie historiciste qui tend à négliger la matérialité textuelle.

C'est bien en réaction à des conjectures censées évaluer les compétences rhétoriques de Mme de Sévigné (à la fois savoir théorique et aptitude pratique) que Christine Noille propose, dans l'article qui clôt le recueil de contributions à titre de bilan et d'ouverture, un changement de perspective radical. Le renouvellement de notre rapport au texte de la Correspondance pour lequel plaide Christine Noille invite à substituer à la quête chimérique de la vérité d'un art d'écrire la mise en œuvre d'un art de lire postulant la capacité du lecteur (autorisé par la lettre du texte) à repérer des dispositifs textuels. Ce qui est en jeu ici, c'est le statut du texte - le texte étant appréhendé comme effet indissociable de la part de la lecture dans son actualisation. L'avantage qu'il y a à penser le texte en termes d'effet actualisé dans et par la lecture est de rompre explicitement avec des questionnements biographiques (projet de l'épistolière, nature du génie sévignéen, genèse de l'écriture...) qui non seulement sont voués à l'échec mais encore éloignent de la description précise du texte. Pour peu que l'on ose, à contrecourant des grilles de lecture majoritaires, s'affranchir de l'«herméneutique de l'intentionnalité », on reconnaîtra que ce genre de questionnements n'apporte rien à la connaissance du texte, "sa cartographie, sa description, son intelligibilité, son efficacité ». Que l'on puisse s'abriter derrière le fait que Mme de Sévigné maîtrisait sans doute les rudiments de la rhétorique (dans ses pratiques de lecture comme d'écriture) " est d'un certain point de vue strictement accessoire : c'est un problème qui ne change rien à la matérialité du texte donné à lire et aux lectures rhétoriciennes qui peuvent en être faites ». Vouloir concentrer l'attention uniquement sur des dispositifs textuels implique en effet d'avoir le courage intellectuel d'affirmer haut et fort que « la validité 
d'une analyse ne dépend pas de sa vraisemblance historique », mais seulement « de la correction avec laquelle elle est effectuée et de sa compatibilité avec le fait du texte ».

À l'issue de ce tour d'horizon des idées reçues qui constituent la toile de fond de la tradition critique dominante, on comprend mieux pourquoi la Correspondance a si longtemps fait les frais d'habitudes herméneutiques propres à en gommer voire à en trahir la dimension textuelle. Récuser les illusions d'inspiration biographique, les mythes esthétiques et les frilosités épistémologiques constituent ainsi trois conditions nécessaires à la mise en œuvre de modes de lecture attentifs aux procédures et aux agencements signifiants. Nécessaires, ces conditions ne sont néanmoins pas suffisantes, puisque pour pouvoir décrire les formes textuelles, encore faut-il pouvoir les repérer au sein d'un tissage qui, nous l'avons dit, échappe en grande partie aux schémas épistolaires canoniques et aux règles discursives répertoriées. D'où la nécessité de poser sur le texte un regard qui, au cours de l'expérience de lecture, soit capable de percevoir et d'identifier des opérateurs formels inégalement visibles.

\section{Les vertus formalisatrices d'une rhétorique de la lecture}

25 Face à un flux textuel qui paraît volontiers sans prise, la mise au jour de techniques de mise en forme implique la reconnaissance de stéréotypes langagiers issus de formations socio-discursives variées - manière de dire que le repérage de dispositifs textuels nécessite de la part du lecteur des aptitudes spécifiques. Comme le souligne Christine Noille dans l'article conclusif, «les dispositifs rhétoriques ou poétiques embrayeurs d'effets sont quelque part dans le texte, mais ils sont rarement des données objectives incontournables (sauf dans le cas où un auteur les sur-inscrit et en quelque sorte les exhibe) ; leur visibilité, leur "remarquabilité", dépend bel et bien du seul lecteur, de sa compétence à les remarquer ». Bref, pour déchiffrer ces dispositifs embrayeurs d'effets, encore faut-il disposer d'outils.

Dans cette quête d'instruments et de modèles de compréhension susceptibles de rendre compte des effets de la Correspondance, il est assurément commode de puiser dans la vaste réserve des savoirs et des compétences partagés par l'élite à laquelle appartenait Mme de Sévigné. Ne nous y trompons pas cependant : s'il y a une pertinence à mobiliser des outils faisant partie de l'épistémè des contemporains de la marquise pour évaluer le fonctionnement et l'impact de procédés textuels, ce n'est pas parce que cela permettrait d'accéder à une vérité historique venant légitimer le travail lectorial et ses manipulations constitutives - un tel besoin de justification s'enracinant dans le confus sentiment de culpabilité causé par la dimension artificielle de toute lecture. Loin de nous l'idée, en optant pour l'utilisation méthodique d'outils contextuels, de défendre un quelconque purisme ayant la prétention d'atteindre la mentalité classique (mentalité qui ne sera jamais que le fruit d'une reconstitution à forte teneur idéologique), voire un quelconque traditionnalisme interdisant de mettre à profit d'autres instruments d'investigation que ceux légués par le Grand Siècle (instruments dont l'usage sera, de toute façon, toujours déformé et réactualisé)! S'il s'avère opportun de déployer des protocoles de lecture inspirés d'usages historiquement attestés, c'est seulement au nom de leur efficacité technique. Autrement dit, dans les opérations de perception et d'intelligibilité des formes textuelles caractéristiques d'une rhétorique de la lecture, les procédures discursives mises à disposition par «la 
bibliothèque » et « l'encyclopédie ${ }^{46}$ " propres au milieu de Mme de Sévigné doivent être envisagées non pas (en amont) comme des techniques d'écriture mais (en aval) comme des formalisations disponibles susceptibles d'être activées à réception par le lecteur.

Qu'il soit possible de décoder des programmes de lisibilité restés inexploités, grâce à un savoir-lire apte à percevoir les effets de lecture induits par le réinvestissement, en régime épistolaire, de patrons associés à certains types de production discursive spécifiques, c'est ce qu'illustrent les quatre contributions centrales. Toutes invitent, à rebours du grand nombre de travaux qui appréhendent la correspondance entre Mme de Sévigné et Mme de Grignan comme un tête-à-tête intime fonctionnant sur le mode d'un huis-clos obsessionnel largement idiolectal, à resituer l'échange épistolaire au sein de tout un ensemble de pratiques discursives communes. L'enjeu est de désenclaver le discours épistolaire au profit de son articulation avec des usages discursifs qui, pour avoir été souvent perdus de vue, n'en constituent pas moins des modèles et des programmes susceptibles d'orienter la démarche de lecture - étant entendu que ces termes de modèles et de programmes, loin de désigner des systèmes normatifs figés prescrivant des recettes artificielles destinées à guider la production, sont à prendre au sens heuristique de grilles d'analyse. Ces usages discursifs dont les conventions régissent un certain nombre d'effets de lecture proviennent de champs variés : il s'agit tantôt de petits genres alors à la mode dans la sphère mondaine, tels que les questions d'amour ou les caractères, qui sous-tendent deux degrés différents de feuilletonisation des récits sévignéens (article de Laure Depretto); tantôt de techniques rhétoriques répertoriées de longue date, telles que l'exemplum, qui permet de rendre compte de la fonctionnalité de nombreux micro-récits factuels de la Correspondance (article de Karine Abiven); tantôt de stratégies d'écriture abondamment cultivées par les romans baroques, tels que les glissements et artifices chronologiques, l'enchaînement d'une ouverture in medias res et d'une analepse, l'enchâssement d'histoires ou encore la formulation d'énigmes, qui compliquent et enrichissent le matériau narratif dont dispose l'épistolière (article de Lise Charles) ; tantôt de rituels constitutifs de la civilité sous l'Ancien Régime, tels que les compliments de condoléance, dont les ressorts pathétiques irriguent les protestations de sympathie maternelles (article de Cécile Lignereux).

Si différentes usages discursifs méritent ainsi d'être mobilisés pour décoder les signaux et les marqueurs textuels qui rendent tel ou tel effet de lecture possible, c'est qu'ils fonctionnent, le temps de la lecture, comme des modèles interprétatifs permettant le repérage et l'analyse de détails formels. L'indéniable fécondité de la démarche qui consiste à mettre au jour les procédures constitutives de la gestion de la lisibilité épistolaire grâce à la sollicitation des habitudes langagières de l'âge classique est riche d'enseignements méthodologiques: pour pouvoir évaluer le rendement des formes textuelles, il peut être utile de les historiciser - et pour cela de se défaire du fétichisme du texte au profit de modélisations fondées sur des cadres et des moules discursifs aptes à fournir d'utiles points de comparaison. S'intéressant non seulement aux effets produits par le texte mais encore à leurs conditions de possibilité, les contributions de Karine Abiven, de Lise Charles, de Laure Depretto et de Cécile Lignereux illustrent ainsi la nécessité, pour rendre compte des dispositifs qui surgissent (potentiellement) à la lecture, de se prémunir contre la tendance qui consiste à présupposer naïvement l'insularité de l'échange épistolaire. 
29 En expérimentant différents dispositifs possibles du texte de la Correspondance, ces quatre contributions engagent tout un système de lecture dont il convient de rappeler les deux principes heuristiques: premièrement, la pratique systématique du séquençage et de la mise en réseau, dans une logique modulaire; deuxièmement, la revendication assumée de la responsabilité du lecteur dans l'apparition de formes et de structures textuelles.

\subsection{La pratique systématique du séquençage et de la mise en réseau}

30 Allant à l'encontre de la conception de la Correspondance comme flux verbal aussi incontrôlable (à la production) qu'intangible (à la réception), l'approche modulaire constitue un instrument d'analyse décisif pour renouveler la description des mécanismes de textualisation. Les lettres de Mme de Sévigné présentant souvent des configurations séquentielles complexes, il s'agit de rendre compte de l'hétérogénéité compositionnelle du discours épistolaire en révélant des formes d'organisation et des schémas configurationnels sous-jacents.

31 Considérer la lettre comme une structure composée de plusieurs séquences précisément isolables, à l'économie et aux modalités propres ${ }^{47}$, ne constitue au demeurant ni une décision arbitraire ni un geste sacrilège tant cette conception fait écho aux pratiques d'écriture, de lecture et d'interprétation des contemporains de Mme de Sévigné. Quatre sortes de considérations nous orientent vers une conception modulaire du texte épistolaire. Premièrement, les manuels épistolographiques, dont le nombre d'éditions atteste la diffusion massive, proposent toutes sortes d'agencements opératoires (certes appelés à être adaptés par les épistoliers en fonction du contexte d'énonciation) qui rendent familières les activités de montage et de hiérarchisation - assemblages, partitions, enchaînements. Deuxièmement, les premiers lecteurs des lettres de Mme de Sévigné n'hésitaient pas à prélever dans ses lettres des unités dont ils percevaient intuitivement l'autonomie (sans le paragraphage ajouté par l'éditeur Roger Duchêne), qu'il s'agisse de destinataires isolant des morceaux choisis pour les lire oralement auprès d'un cercle de proches ${ }^{48}$ ou d'éditeurs découpant des passages jugés dignes de figurer dans des anthologies. Troisièmement, l'écriture épistolaire accueille et intègre avec souplesse (une souplesse irréductible au goût des contemporains de Mme de Sévigné pour l'esthétique fragmentaire, à l'œuvre notamment dans les maximes, portraits, anecdotes ou conversations) une myriade de formes, qu'elle choisisse ou non de les infléchir, de les croiser ou de les décliner - tant il est vrai que la «polygraphie épistolaire» favorise les «tentatives de brouillages des codes littéraires existants », « la contamination des modèles préétablis » et «les efforts de recomposition qui récusent la solidité théorique des genres canoniques ${ }^{49}$ ». Enfin, il semblerait même que nous ayons la caution de l'auteur pour fractionner ses lettres, dans la mesure où Mme de Sévigné ne cesse de pointer des entités textuelles indépendantes au moyen de toutes sortes de dénominations métadiscursives plus ou moins pittoresques (" causerie », « chapitre », « réflexion », « radoterie »...). Voilà plus de données contextuelles qu'il n'en faut pour apaiser la mauvaise conscience du lecteur qui aurait du mal à assumer ses opérations (plus ou moins faciles, plus ou moins discrètes) de découpage ! 

baliser des blocs textuels dotés d'un fonctionnement interne plus ou moins préformaté, donne les moyens d'éviter un double écueil dans le repérage, l'identification et l'interprétation des dispositifs textuels: d'un côté (si l'échelle est trop réduite et cantonne le regard au fonctionnement linguistique d'un fait), atomiser le texte en déconnectant artificiellement le phénomène étudié de son contexte d'apparition; de l'autre (si l'échelle est trop grande et élargit le regard aux constantes esthétiques de la correspondance), perdre de vue le détail du texte en diluant le phénomène étudié dans une combinatoire d'invariants stylistiques. C'est par opposition à ces deux échelles textuelles (unités trop réduites, unités trop vastes) qu'il s'agit de mettre au jour des unités d'analyse intermédiaires, selon une logique modulaire. Dans cette entreprise de séquençage destinée à délimiter des unités au niveau desquelles il devient possible de rendre compte de la fonctionnalité convergente d'embrayeurs de structuration, il s'agit de ne pas se laisser dérouter par deux phénomènes qui peuvent à première vue gêner les opérations de formalisation et de modélisation.

D'une part, l'inégalité des étendues textuelles considérées doit être tenue pour négligeable. Certes, les unités textuelles sont de tailles extrêmement variables: une anecdote à caractère exemplaire (Karine Abiven), un récit informé par la poétique romanesque (Lise Charles), un épisode de feuilleton narratif (Laure Depretto) ou un compliment de condoléance (Cécile Lignereux) peut tantôt se réduire à quelques phrases, tantôt s'étendre sur de nombreuses lignes. De même que la question des variations d'amplitude des masses textuelles n'était assurément pas prioritaire dans les exercices de lecture pratiqués jadis dans les classes de rhétorique, elle s'avère, dans le protocole de lecture fondé sur des faits de compositionnalité, tout à fait secondaire. Souvenons-nous que pour les commentateurs comme pour les professeurs d'Ancien Régime (notamment le jésuite Joseph Jouvancy, qui, dans son fameux manuel maintes fois réédité, procède pour ses élèves au balisage d'une trentaine de lettres de Cicéron ${ }^{50}$ ), peu importe la longueur de la séquence, pourvu qu'y converge un faisceau d'indices spécifiques.

D'autre part, il ne faut pas se laisser impressionner par le fait que dans une séquence, tous les indices virtuellement embrayeurs ne sont pas actualisés en même temps. S'il serait effectivement plus facile et plus rassurant, pour le lecteur soucieux d'étiqueter et d'empaqueter des segments textuels, de n'avoir affaire qu'à des configurations pleinement stabilisées et donc formellement identiques, force est de constater que le degré de canonicité d'une séquence varie considérablement. C'est ainsi que décrites à l'aune de différents «types de relations macro-sémantiques mémorisées par imprégnation culturelle [...] et transformées en schémas de reconnaissance et de structuration de l'information textuelle ${ }^{51}$ ", les séquences isolables au fil du texte sont à concevoir moins comme des unités organisées selon des opérations aussi rigides qu'immuables que comme des agencements qui, s'ils sont effectivement typés (et donc reconnaissable à la lecture), reposent sur la sollicitation occasionnelle de procédures facultatives stockées dans la mémoire discursive commune. Il suffit qu'un nombre réduit de propriétés propres à certains usages discursifs soit actualisé pour que se déclenche, au cours de la lecture, le mécanisme de structuration, de modélisation et d'identification de la séquence. Ainsi en va-t-il des récits à portée exemplaire (examinés par Karine Abiven), dont les cinq techniques de mise en forme qui en provoquent la reconnaissance ne se cumulent pas nécessairement — signalétique métalinguistique, 
comparaison d'essence narrative, puissance imageante du récit, trajet cognitif fondé sur l'abduction, tropisme ludique; des épisodes narratifs prenant part au sein d'un macro-récit qui se diffuse sur plusieurs lettres (qu'analyse Laure Depretto), dont les indices de feuilletonisation peuvent plus ou moins se conjuguer - affectation d'un identifiant, emploi de balises temporelles, élaboration de configurations hypothétiques et provisoires; des compliments de condoléance (étudiés par Cécile Lignereux), qui n'actualisent généralement pas de manière concomitante les quatre procédés les plus usités en pareille circonstance - isotopie de la compassion, modalité exclamative, constructions à verbe support, périphrases verbales factitives.

Quoi qu'il en soit de la densité du marquage technique permettant le balisage, l'identification puis la mise en réseau d'une séquence textuelle, on mesure le rôle décisif, dans l'exploration des configurations compositionnelles, de la manière de lire. Pour aller vite, disons qu'une séquence textuelle n'est jamais donnée : elle est toujours construite - tout avènement de séquence reposant sur un certain nombre d'opérations lectoriales qui, si elles sont autorisées par la matérialité du texte, n'en fonctionnent pas moins comme des activités de structuration et de modélisation. C'est donc non seulement à comprendre les procédures lectoriales mais aussi et surtout à les assumer que convie une rhétorique comprise comme un art de lire qui ne cesse de faire retour sur le processus de formalisation qui lui est inhérent.

\subsection{La responsabilité du lecteur dans l'apparition de formes et de structures textuelles}

Déployant, à l'instar des exercices pédagogiques des siècles passés, un art de lire conçu comme un art d'organiser des masses textuelles, de repérer des configurations et d'en proposer des modélisations, les quatre contributions centrales de ce numéro illustrent de manière décisive le rôle du lecteur dans l'avènement de formes et de structures textuelles. Loin d'aboutir à des descriptions d'agencements discursifs aussi objectifs qu'irréfutables, elles ne font que proposer des expériences de lecture. Abandonnant toute prétention à " entrer par la bonne porte dans le monde sévignéen ${ }^{52}$ ", ces articles cultivent en effet des gestes techniques explicitement tournés vers la pratique de la lecture: repérage et autonomisation d'unités relativement indépendantes qui se découpent sur le fond du tissu épistolaire ; mise en série des séquences par enquête sur les procédés standardisés et leurs variantes; description de leur fonctionnement interne grâce à la confrontation avec différents modèles formels extérieurs ; appréciation de leurs effets de bornage, d'enchaînement et de hiérarchisation au sein de la dynamique textuelle. Parce qu'ils aboutissent à la construction de sous-ensembles requérant chacun des procédures d'investigation spécifiques, ces gestes de structuration du texte ont comme effet d'attirer l'attention sur des formes possibles - à une échelle précisément localisée. Compris comme une expérimentation susceptible de construire la lisibilité de dispositifs textuels, un tel art de lire n'affiche en effet pas d'autre ambition que celle de formuler des hypothèses à la fois d'ordre formel (il s'agit d'isoler des formes plus ou moins autonomes) et d'ordre fonctionnel (il s'agit d'expliquer la fonctionnalité de procédures, c'est-à-dire leur cohérence et leur efficacité) - ce qui revient à dire que tout est affaire de réception.

Tester la validité de modèles de compréhension, c'est-à-dire proposer, sur le mode d'hypothèses, des interprétations possibles du texte : telle est la démarche commune 
aux contributions de Karine Abiven, de Lise Charles, de Laure Depretto et de Cécile Lignereux. Partant du principe qu'un texte se contente d'offrir au lecteur des potentialités (que celui-ci exploitera ou non, en fonction de ses compétences), ces contributions ne livrent jamais que des repérages et des identifications qui visent seulement à être le plus rentables possible pour rendre compte d'effets configurationnels. Il serait en effet utopique de croire que l'opération lectoriale de mise en forme de masses discursives par des modèles extérieurs aboutit à une prise en charge des séquences isolées et sérialisées qui soit à la fois exhaustive, stabilisée et exclusive. Disons que les formalisations proposées sont relativement satisfaisantes dans la mesure où elles parviennent à intégrer un maximum des procédés convergents caractéristiques des séquences décrites. Certes, les modélisations obtenues d'après des pratiques rhétoriques (l'exemplum pour Karine Abiven), des stratégies romanesques (le suspens et la mise en intrigue pour Lise Charles), des filtres cognitifs (la feuilletonisation pour Laure Depretto) ou des codes du savoir-vivre (la condoléance pour Cécile Lignereux) sont aussi opératoires que possible puisqu'elles permettent, adossées à de solides indices textuels, de décrire l'économie globale de séquences dont elles démontent efficacement les procédés solidaires. Néanmoins, c'est le lot de toute modélisation, aussi motivée et raisonnée soit-elle, que de ne pas pouvoir prendre en charge la totalité des phénomènes agencés au sein d'une séquence : puisque l'analyse rhétorique procède en s'attachant aux procédés typiques, c'est-à-dire aux régularités permettant de diagnostiquer de l'exemplaire, elle s'avère vouée à laisser de côté un nombre plus ou moins grand de phénomènes irréductibles à la modélisation textuelle retenue (après avoir été méthodiquement conçue, éprouvée, amendée et validée). Que toute entreprise de formalisation se heurte à des limites n'entame pour autant ni la légitimité ni la rigueur d'une démarche qui a pour tâche de décrire l'opérativité de séquences étudiées du point de vue du commun et du standard. Aussi déstabilisant que cela puisse paraître, la prise en compte des points de résistance du texte (qui contrarient voire contredisent les formatages envisagés) constitue le passage obligé de toute tentative de modélisation textuelle, comme en témoigne la rhétorique de la lecture que pratiquent par exemple, quoique dans des perspectives différentes, Michel Charles (qui nous a sensibilisés aux dysfonctionnements venant compliquer les montages textuels ${ }^{53}$ ), Francis Goyet (qui nous a familiarisés avec les divergences voire les incohérences qui ne manquent pas de surgir sitôt que l'on teste l'applicabilité d'un modèle explicatif ${ }^{54}$ ) ou Christine Noille (qui nous a accoutumés à la possibilité de proposer plusieurs formatages pour un même texte ${ }^{55}$ ).

Insistons-y : la gestion des éléments textuels qui viennent enrayer (provisoirement ou non) le protocole qui s'attache à mettre en forme le texte fait partie intégrante de la dynamique intellectuelle propre à la rhétorique de la lecture - mettre en forme le texte ne signifiant en aucun cas succomber à la tentation de le lisser au point d'en gauchir ou d'en nier les éléments impossibles à résorber dans le modèle de description envisagé. On aurait tort de se priver de modèles de description opératoires sous prétexte que certains des éléments qui se combinent au sein des séquences analysées résistent et s'avèrent inassimilables au modèle de fonctionnement retenu. Ajoutons d'ailleurs qu'il ne s'agit pas de niveler les faits textuels en niant les points de fragilité inhérents à toute formalisation : évidemment que la logique exemplaire des anecdotes de Mme de Sévigné est souvent brouillée par la facétie ou le badinage galant (Karine Abiven); que les stratégies romanesques perceptibles dans la Correspondance subissent une extrême miniaturisation (Lise Charles); que les conditions requises pour que 
s'opère la constitution d'un feuilleton lors de la lecture de nouvelles consacrées à une même personne sont inégalement satisfaites (Laure Derpetto); que les condoléances adressées à Mme de Grignan ne recourent ni à la disposition fixe, ni aux lieux communs, ni aux précautions oratoires dont l'usage est alors topique. Il n'en reste pas moins que ces grilles de formalisation ont le mérite de rendre compte de l'économie globale de blocs textuels restés jusque-là informes, c'est-à-dire illisibles. C'est pourquoi il paraît raisonnable de penser qu'il est fécond d'inventer de nouveaux modèles de description aptes à mettre au jour de nouveaux programme de lisibilité. Simplement, il convient d'être bien au clair sur le partage des tâches qui s'opère dans et par la lecture.

Appréhender le texte par le biais de l'intervention du lecteur (plus ou moins formé, plus ou moins informé) dans l'actualisation de tel ou tel axe de lisibilité entraîne des conséquences décisives pour la pratique de la lecture et pour la définition du statut du texte. À partir du moment où l'on admet que l'établissement de telle ou telle modélisation du texte dépend de la seule capacité du lecteur à repérer et à exploiter les éléments qui autorisent à proposer cette version possible du texte, c'est toute la relation du lecteur au texte qui est à repenser - un texte conçu non plus comme un système figé existant en soi et recelant des procédés en attente d'être repérés et expliqués (selon l'optique scolaire traditionnelle, que Michel Charles désigne comme culture du "commentaire») mais comme un montage riche de possibilités et indissociable de la dynamique lectoriale, qui construit la lisibilité du texte en octroyant à certains éléments la fonction d'embrayeurs de structuration (dans la perspective que Michel Charles qualifie de "rhétorique ») ${ }^{56}$. Car l'important est bien là, dans le rapport au texte. Puisque le lecteur, loin de constater passivement l'existence de procédés qui n'attendraient que d'être perçus, motive des formes, active des structures et actualise des modèles, il convient de s'interroger sur la manière dont certains éléments textuels sont susceptibles d'être perçus, au cours de l'expérience de lecture, comme des opérateurs de mise en forme. Une fois encore, voilà qui force à reconnaître la responsabilité du lecteur - une responsabilité à la fois vertigineuse et canalisée. Vertigineuse, puisque décrire un texte en l'ordonnant aboutit à décider de ses formes possibles, un même texte autorisant plusieurs schémas d'explication. Canalisée, dans la mesure où la description des formes textuelles se fonde sur des possibilités offertes par le texte. En définitive, si le texte comprend bien une part d'instabilité voire de contingence, celle-ci est toutefois relativisée par le fait que le travail lectorial se contente de tirer parti, aussi rigoureusement que possible ${ }^{57}$, de données textuelles. $\mathrm{Ni}$ anarchique ni arbitraire, la décision d'octroyer à certains procédés un rôle de marqueur ne consiste jamais qu'en l'actualisation de certaines potentialités du texte. De fait, « le repérage d'une forme du texte va dépendre de la compétence du lecteur à repérer non seulement des indices de mise en forme inscrits (plus ou moins discrètement ici) par l'auteur mais également à motiver des éléments textuels comme embrayeurs (indicateurs) de forme, à investir certains dispositifs textuels d'un fonctionnement structurant ${ }^{58} »$.

Ce qui veut dire que «chaque lecteur pourra activer (actualiser) une forme du texte différente ${ }^{59}$ ». Alors qu'il est possible de déceler une teneur morale dans un certain nombre des micro-récits de Mme de Sévigné, rien n'oblige le lecteur à actualiser le modèle de l'exemplum : "un même récit peut être interprété comme le vecteur d'une signification qui le dépasse (morale, exhortation indirecte, etc.) ou comme une pièce du jeu divertissant qu'est, aussi, la correspondance » (article de Karine Abiven). Alors qu'il est possible d'éprouver que les stratégies de suspens et de mise en intrigue à l'œuvre 
dans certains récits font signe vers la fiction, le lecteur n'est en aucun cas contraint d'activer les ouvertures fictionnelles que suggèrent les affinités entre ces stratégies et la poétique du roman baroque, surtout s'il valorise les spécificités de la narration épistolaire en termes de matériau, de structuration du récit et d'énonciation (article de Lise Charles). Alors qu'il est possible de repérer entre certaines séquences informatives "différentes passerelles [...] d'une lettre à l'autre, soit dès la première lecture, parce que Sévigné signale elle-même une suite à venir ou qu'elle rappelle les faits, soit à la relecture, lorsqu'il est possible d'apercevoir rétrospectivement la présence d'une trame liant plusieurs lettres", le lecteur peut tout à fait ignorer de tels "effets de continuité » : il suffit pour cela qu'il se contente de lire chacun des passages concernant une même personne non pas comme des séquences éclatées d'un macro-récit se déployant sur plusieurs lettres mais comme de simples nouvelles ponctuelles ne dépassant pas le cadre de la lettre unique (article de Laure Depretto). Alors qu'il est possible de mettre en série les assurances de sympathie maternelles sur le modèle des compliments de condoléance alors abondamment pratiqués, le lecteur peut les intégrer à d'autres dynamiques discursives - plaintes, exhortations, reproches ou requêtes (article de Cécile Lignereux). On l'aura compris, lorsque plusieurs modèles de schématisation cautionnés par la matérialité textuelle s'avèrent également efficaces, c'est au lecteur de décider de valider l'un ou l'autre, c'est-à-dire de privilégier l'un des textes possibles ${ }^{60}$. Face aux embranchements, bifurcations et points de bascule offerts le temps de la lecture, le lecteur peut en effet choisir de privilégier tel élément textuel et d'ignorer tel autre: tout dépend du système de lecture adopté ${ }^{61}$. Aux potentialités offertes par le texte, le lecteur répond toujours en fonction de son horizon d'attente. Libre à lui de gérer ses relevés formels (en motivant tel élément, en désactivant tel autre) au gré du modèle de lecture qu'il décide d'actualiser - une décision qui, hâtonsnous de le préciser, relève non plus de la rhétorique mais de l'interprétation ${ }^{62}$.

Au-delà du renouvellement des modes de lecture et d'interprétation des lettres de Mme de Sévigné, l'enjeu est donc bien celui d'une réflexion sur les différentes relations possibles du lecteur au texte épistolaire. Parce qu'elles déploient «non pas un art de bien "voir" des dispositifs latents et en attente d'être vus, mais un art de formater le fil du texte à partir d'un certain nombre de dispositifs types, un art de motiver dans le texte un certain nombre d'énoncés comme embrayeurs de structuration et de modélisation, bref, redisons-le, un art de construire sa lisibilité en composant sa forme ${ }^{63}$ ", les contributions réunies constituent d'authentiques exercices de rhétorique. Se présentant explicitement non pas comme des "descriptions de procédés d'écriture " mais comme " différents types de questions à poser et différents parcours à effectuer ${ }^{64}$ ", elles invitent à faire l'économie des débats récurrents autour d'enjeux psychologiques (la personnalité de l'auteur, ses affects et ses motivations), anthropologiques (l'écriture épistolaire comme pratique ritualisée reposant sur des mécanismes d'appropriation) ou esthétiques (la fabrication d'un chef-d'œuvre incarnant les valeurs du classicisme) au profit de l'expérimentation d'hypothèses de modélisations - substituant la description à l'interprétation, l'observation technique à l'appréciation littéraire, l'enquête sur l'exemplaire à la recherche du singulier, l'explication d'effets du texte à des spéculations sur sa genèse.

En déplaçant le regard du côté des dispositifs textuels de la Correspondance, c'est-à-dire en l'appréhendant comme système descriptible, ces contributions illustrent à la fois l'humilité et l'ambition d'une rhétorique de la lecture qui se contente volontairement 
de repérer des formes et des modèles possibles: son humilité d'abord, puisqu'elle reconnaît ne pas avoir les moyens de se prononcer sur des intentions et des finalités; son ambition ensuite, dans la mesure où elle invente de nouveaux outils d'analyse, de nouveaux modèles de description, de nouveaux programmes de lisibilité. À systématiser un tel protocole de lecture, peut-être certaines images d'Epinal complaisamment véhiculées au fil des siècles sur l'aimable marquise se trouvent-elles écornées - dépoussiérées en tout cas. Il n'est toutefois pas impossible que l'on puisse préférer, plutôt que de perpétuer naïvement le réflexe scolaire qui consiste à justifier la tradition hagiographique qui entoure l'un des monuments de notre patrimoine littéraire, essayer sur le texte différents " points de vue variables et dont la variabilité est sinon réglée, du moins contrôlée par la rhétorique ${ }^{65} »$.

\section{NOTES}

1. G. Genette, Fiction et diction, Paris, Seuil, coll. « Poétique », 1991, p. 7.

2. Ibid., p. 7-8.

3. M. Charles, Rhétorique de la lecture, Paris, Seuil, coll. « Poétique », 1977, p. 118-119.

4. Ibid., p. 63.

5. Ibid., p. 247.

6. Nous renvoyons au recensement exhaustif des commentaires et études sur Mme de Sévigné du XVIII ${ }^{\mathrm{e}}$ siècle aux années 1970 effectué par Fr. Nies, Les Lettres de Mme de Sévigné. Conventions du genre et sociologie des publics [1972], Paris, Champion, coll. « Lumière classique », 2001, p. 319-380. Pour une bibliographie actualisée, on se reportera à C. Lignereux (dir.), La première année de correspondance entre Mme de Sévigné et Mme de Grignan, Paris, Classiques Garnier, coll. « Mémoires et correspondances ", 2012, p. 315-332.

7. Que les optiques textuelles, notamment dans le prolongement des articles novateurs de B. Bray et d'I. Landy-Houillon, soient de plus en plus fréquemment convoquées pour étudier les lettres de Mme de Sévigné, c'est ce dont témoignent les treize études réunies dans C. Lignereux (dir.), Lectures de Mme de Sévigné. Les lettres de 1671, Rennes, PUR, 2012 - qu'il s'agisse d'approches rhétoriques (Chr. Noille, J.-Y.Vialleton, C. Lignereux), poétiques (A. Brunn, S. Gruffat, L. Depretto, C. Pagani-Naudet), interdiscursives (Ch. Barbillon, É. Tourrette, C. Cagnat-Debœuf) ou stylistiques (S. Macé, F. Boissieras, A.-M. Garagnon).

8. Sur les soubassements, chronologiquement contrastés, d'une postérité lourde de présupposés idéologiques, voir Fr. Nies, Les Lettres de Mme de Sévigné. Conventions du genre et sociologie des publics, op. cit. Répertoriant les lectures qui ont été faites des lettres de Mme de Sévigné, l'auteur s'attache à en analyser les fondements - à savoir les goûts et les attentes des publics successifs essentiellement jusqu'aux années 1960. Du même auteur, voir également le panorama des lectures de Mme de Sévigné qui ont été faites du début des années 1970 jusqu’à nos jours, « Déclin de l'éducation littéraire, critique inopérante?», Europe, n 801-802, janvier-février 1996, p. 123-132.

9. Sur la succession de hasards qui a présidé à la métamorphose d'une correspondance privée en chef-d'œuvre de la littérature, on se reportera R. Duchêne, «Du destinataire au public, ou les métamorphoses d'une correspondance privée », Revue d'Histoire Littéraire de la France, 1976, n¹, p. 29-46. 
10. «La lecture est une expérience et se trouve, en tant que telle, soumise à un ensemble de variables qui ne peuvent a priori relever de la théorie de la littérature. Dans le grand jeu des interprétations, les forces du désir et les tensions de l'idéologie ont un rôle décisif. Il reste que ce jeu n'est possible que dans la mesure où les textes le permettent. Cela ne signifie pas qu'un texte autorise n'importe quelle lecture, mais simplement qu'il est marqué d'une essentielle précarité, qu'il a lui-même du jeu. ", M. Charles, Rhétorique de la lecture, op. cit., p. 9.

11. A. Viala, «Un jeu d'images: amateur, mondaine, écrivain?», Europe, $n^{\circ} 801-802$, janvierfévrier 1996, p. 57-68.

12. Nous renvoyons aux prises de position antagonistes de Bernard Bray («Quelques aspects du système épistolaire de Mme de Sévigné » [1969], repris dans Épistoliers de l'âge classique, L'art de la correspondance chez Mme de Sévigné et quelques prédécesseurs, contemporains et héritiers, Tübingen, Narr, coll. «Études littéraires françaises ", 2007, p. 245-259) et de Roger Duchêne («Réalité vécue et réussite littéraire : le statut particulier de la lettre » [1971], repris dans Écrire au temps de Mme de Sévigné. Lettres et texte littéraire, Paris, Vrin, 1982, p. 11-28), ainsi qu'au résumé que propose Bernard Beugnot du contenu et des enjeux de cette polémique ("Débats autour du genre épistolaire. Réalité et écriture », Revue d'Histoire Littéraire de la France, mars-avril 1974, p. 195-202). 13. Nous renvoyons à la mise au point de Roger Duchêne dans sa « Note sur le texte » (Mme de Sévigné, Correspondance, éd. R. Duchêne, Paris, Gallimard, coll. «Bibliothèque de la Pléiade », 1972-1978, tome I, p. 755-830).

14. Chr. Noille, «Les lettres de Sévigné sont-elles informes ? Éléments pour une rhétorique de la disposition ", dans C. Lignereux (dir.), Lectures de Mme de Sévigné. Les lettres de 1671, op. cit., p. 19-47.

15. Les références aux lettres de Mme de Sévigné mentionnent la date de la lettre et sa pagination (tome et page) dans l'édition de référence : Correspondance, éd. R. Duchêne, op.cit. Nous ne précisons le destinataire que lorsqu'il ne s'agit pas de Mme de Grignan.

16. Nous renvoyons successivement aux analyses de K. Abiven, "Si vous n'aimez ces traits-là, dites donc mieux". Quelques outils pour l'analyse textuelle de l'anecdote chez Mme de Sévigné », L'Information grammaticale, $\mathrm{n}^{\circ} 136,2013$, p. 3-6; de C. Lignereux, "Vers une typologie des anecdotes galantes de Mme de Sévigné », Littératures classiques, $\mathrm{n}^{\circ} 78$, «Écritures de l'actualité [XVI ${ }^{\mathrm{e}}$-XVIII ${ }^{\mathrm{e}}$ siècles] », K. Abiven et L. Depretto (dir.), 2012, p. 97-109; de L. Depretto, L'histoire du jour. Informer et raconter dans la Correspondance de Mme de Sévigné, Paris, Classiques Garnier, à paraître.

17. Ch. Noille, «Les lettres de Sévigné sont-elles informes ? Éléments pour une rhétorique de la disposition ", art. cit.

18. Nous faisons allusion à l'ouvrage de R. Duchêne, Mme de Sévigné ou la chance d'être femme, Paris, Fayard, 2002.

19. « Entre les lettres des doctes d'avant la poste et des mondains d'après la poste, il n'y a en fait pas de filiation, ni même de lien, pour la simple raison que les nouveaux épistoliers ignoraient jusqu'à l'existence de leurs illustres prédécesseurs. Je ne vois pas Mme de Sévigné avec un Du Tronchet à la main. Encore moins avec un De Conscribendis Epistolis. ", R. Duchêne, Mme de Sévigné et la lettre d'amour [1970], Paris, Klincksieck, coll. « Bibliothèque de l'âge classique », 1992, p. XIV.

20. Ibid., p. 77-86.

21. Ibid., p. 100.

22. "Que pouvaient apporter à Mme de Sévigné les manuels épistolaires et les recueils ? Dans les volumes de Rosset, de La Serre ou de Faret, dans les Lettres de Balzac, de Malherbe ou de Lannel, dans les Lettres de dames de Duboscq, ou plus tard, dans celles de Grenaille, c'était toujours la même éloquence, la même application des principes de la rhétorique dans une lettre conçue comme l'illustration d'un genre, non comme la réussite spontanée d'une correspondance naturelle ", ibid., p. 99. 
23. Répertoriant les promoteurs de l'«idée d'une épistolarité féminine de qualité » (Duboscq, Cotin, Pinchêne, La Bruyère), Roger Duchêne commente ainsi leur idéal épistolaire: «La créativité féminine n'a pas été étouffée comme celle des hommes par un long et pénible apprentissage de la manière dont il faut écrire. Elles ne sont pas prisonnières des règles de la disposition [...]. Heureuses femmes, qui n'ont appris ni à écrire ni à composer ! Heureuses femmes qui sont, comme Agnès, riches de leur ignorance! », ibid., p. IX.

24. Nous renvoyons aux articles de B. Bray : «Quelques aspects du système épistolaire de Mme de Sévigné » [1969], art. cit. ; « Le style épistolaire : la leçon de Mme de Sévigné » [1996], repris dans Épistoliers de l'âge classique, op. cit., p. 317-330.

25. Voir C. Lignereux, «La déformalisation du dialogue épistolaire dans les lettres de Mme de Sévigné », Littératures classiques, $\mathrm{n}^{\circ} 71$, "L'épistolaire au XVII ${ }^{\mathrm{e}}$ siècle », G. Ferreyrolles (dir.), printemps 2010, p. 113-128.

26. J.-Y. Vialleton, «Écriture épistolaire et rhétorique: Aristote, ancêtre de la marquise de Sévigné ", dans C. Lignereux (dir.), Lectures de Mme de Sévigné, op. cit., p. 58-61.

27. Nous renvoyons successivement à nos articles: "Les offres de service à Mme de Grignan. Enquête sur l'acclimatation familière de préconstruits rhétoriques ", dans C. Lignereux (dir.), Lectures de Mme de Sévigné, op. cit., p. 67-81; «L'exagération de la faute : une technique propre à la lettre de remontrance", Exercices de rhétorique [En ligne], 4|2014, URL: http:// rhetorique.revues.org/349; «D'un sous-genre épistolaire à sa mise en œuvre en contexte familier : l'exhortation ", à paraître dans É. Gavoille et F. Guillaumont (dir.), Conseiller, diriger par lettre (Epistulae antiquae IX), Tours, Presses universitaires François-Rabelais, coll. « Perspectives littéraires ».

28. Pour une récente mise au point sur la signification et les enjeux de ces notions, on se reportera à A.Génetiot, Le Classicisme, op. cit., chap. I de la cinquième partie, "Naturel et agréments ", p. 377-413. On lira notamment les pages consacrées à la naïveté (ibid., p. 377-383) et à la négligence (ibid., p. 387-394).

29. Sur les présupposés culturels et les enjeux idéologiques de la croyance, rendue fameuse par le jugement de La Bruyère, en une supériorité des femmes en matière épistolaire, voir les mises au point de Fr. Nies, «Un genre féminin?», Revue d'Histoire Littéraire de la France, novembredécembre 1978, p. 994-1003; de Chr. Planté, «Introduction » à Ch. Planté (dir.), L'Épistolaire, un genre féminin?, Paris, Champion, coll. «Varia », 1998, p. 11-24 ; d'I. Landy-Houillon, « Le féminin vu par les hommes. L'exemple des Treize lettres amoureuses de Boursault » [1998], repris dans Entre philologie et linguistique. Approches de la langue classique, Paris, Classiques Garnier, coll. « Lire le XVII siècle ", 2012, p. 307-320.

30. Nous empruntons l'expression de R. Duchêne, « Réalité vécue et réussite littéraire : le statut particulier de la lettre » [1971], repris dans R. Duchêne, Écrire au temps de Mme de Sévigné, op. cit., p. 11-28.

31. M. Fumaroli, «Genèse de l'épistolographie classique : rhétorique humaniste de la lettre, de Pétrarque à Juste-Lipse »[1978], repris dans La Diplomatie de l'esprit, Paris, Hermann, coll. «Savoir : Lettres », 1998, p. 168.

32. Nombreux sont les recueils de lettres et les manuels d'art épistolaire à prôner une discrétion stylistique qui n'équivaut pas pour autant à battre en brèche le paradigme de l'«éloquence ». C'est ainsi qu'après avoir souligné « les difficultez qui se rencontrent à reüssir en un caractére si delicat, qui pour estre moins élevé, n'en a pas des lois moins étroites ny moins sevéres » et après avoir rappelé la nécessité de « joindre tout à la fois la simplicité à la délicatesse, \& la douceur à la subtilité », André-Louis Personne insiste sur le fait que «l'éloquence [...] n'est pas bannie des entretiens particuliers » et que tout est affaire de dosages et de proportions : " Mais c'est à dire seulement que ce genre de discours ne reçoit pas les grands mouvements des harangues; que ses beautez sont des beautez plus douces et plus tranquiles, \& qu'en un mot, ce n'est pas dans ces bornes étroites, que l'éloquence se répand en fleuves et en torrens ; mais qu'elle n'y coule que par 
de petits canaux, \& qu'elle laisse toujours beaucoup plus de choses à dire, qu'elle n'en dit. ", Lettres et billets, En tous les genres d'écrire..., Paris, Laurens Raveneau, 1662, "Préface sur le caractère du style épistolaire ", s. p.

33. C. Lignereux, «Scénographies mondaines et réglages polyphoniques dans les lettres de Mme de Sévigné : l'exemple des compliments", dans C. Lignereux et J. Piat (dir.), Une langue à soi. Propositions, Pessac, Presses Universitaires de Bordeaux, coll. «Stylistique et Poétique », 2009, p. 139-158.

34. C. Lignereux, «Défense et illustration des valeurs de Tendre dans les lettres de Mme de Sévigné », Cahiers de narratologie, $\mathrm{n}^{\circ} 10$, «Valeurs et correspondance », A. Tassel (dir.), 2010, p. 13-32.

35. C. Lignereux, «L'inscription des larmes dans les lettres de Mme de Sévigné : tentations élégiaques et art de plaire épistolaire ", Littératures classiques, $\mathrm{n}^{\circ} 62$, «Le Langage des larmes aux siècles classiques", A. Cron et C. Lignereux (dir.), été 2007, p. 79-91; et "Les modulations des aveux de tendresse dans les lettres de 1671 à Mme de Grignan », Styles, genres, auteurs, ${ }^{\circ} 12$, A. Gautier et S. Hériché-Pradeau (dir.), Paris, PUPS, 2012, p. 53-67.

36. C. Lignereux, «Du fait de langue à l'effet de style: les zeugmes syntaxiques de Mme de Sévigné », dans L. Himy-Piéri, J.-F. Castille et L. Bougault (dir.), Le Style, découpeur de réel, Rennes, PUR, coll. « Interférences », 2014, p. 139-150.

37. C. Lignereux, "Les mots de l'idolâtrie dans les lettres de Mme de Sévigné ", dans M. Daumas (dir.), Amour divin, amour mondain dans les écrits du for privé de la fin du Moyen Âge à 1914, Pau, Cairn, 2011, p. 203-219; et « Pour une approche argumentative des binômes synonymiques dans les lettres de Mme de Sévigné ", dans Fr. Berlan (dir.), La Synonymie, Paris, PUPS, 2012, p. 415-424.

38. Nathalie Freidel a proposé un état des lieux concernant la présence d'éléments rhétoriques dans la Correspondance (La Conquête de l'intime. Public et privé dans la Correspondance de Madame de Sévigné, Paris, Champion, coll. "Lumière classique », 2009, p. 668-681), au terme duquel elle conclut que « la rhétorique sévignéenne » est « une manière unique d'articuler les fragments de la rhétorique classique autour de concepts subjectifs », et que « c'est ce mélange indéfinissable de conformité et d'originalité, de conventions et d'inventions, qui nous semble faire la saveur particulière du style sévignéen » (ibid., p. 679).

39. R. Duchêne, Mme de Sévigné et la lettre d'amour, op. cit., p. 101.

40. On aura reconnu la distinction proposée par Roger Duchêne : «Pour clarifier les idées, nous appellerons épistolier celui qui ne tient pas compte de l'existence du public et auteur épistolaire celui qui, au contraire, se soucie plus d'un public éventuel que de celui à qui il est censé écrire. Les lettres du second appartiennent, par définition, à la littérature épistolaire [...]. Les lettres de l'épistolier au contraire ne s'inscrivent pas spontanément dans le genre épistolaire, dont il peut ignorer ou mépriser les lois [...]. », « Réalité vécue et réussite littéraire : le statut particulier de la lettre ", art. cit., p. 11-12. Pour une mise en perspective socio-historique de cette distinction, voir J.-G. Altman, « Espace public, espace privé : la politique de la publication de lettres sous l'ancien régime ", Revue Belge de Philologie et d'Histoire, LXX, 3, «Le Genre épistolaire », Ch. Delcourt (dir.), 1992, p. 620.

41. R. Duchêne, Mme de Sévigné et la lettre d'amour, op. cit., p. 264-269.

42. Roger Duchêne, "Une grande dame et la rhétorique: Madame de Sévigné et le P. Le Bossu » [1977], repris dans R. Duchêne, Écrire au temps de Mme de Sévigné, op. cit., p. 282-283.

43. Ch. Noille, «Les lettres de Sévigné sont-elles informes? Éléments pour une rhétorique de la disposition ", art. cit., p. 26.

44. Tel est le bilan que formule ici même Ch. Noille dans son article intitulé "Brève notice d'histoire littéraire : la question de la rhétoricité sévignéenne ».

45. Citons notamment la position de Bernard Bray : " Il est à peine besoin de recourir au traité de René Bary ou de faire l'inventaire des relations de Mme de Sévigné avec les PP. Bouhours et 
Rapin, pour comprendre que celle-ci appliquait d'instinct et avec la plus grande aisance, guidée pourtant par ses lectures et sa pratique du monde, des règles que les théoriciens de la rhétorique codifiaient avec quelque pesanteur. ", « Mme de Sévigné et l'art de la narration » [1988], repris dans Épistoliers de l'âge classique, op. cit., p. 293.

46. Nous reprenons la distinction conceptuelle que fait Ch. Noille, dans la conclusion de la première partie de son article, entre " une encyclopédie des formes remarquables ", c'est-à-dire " une connaissance des formes remarquées et identifiées par l'analyse rhétorique et poétique ", et « une bibliothèque, la mémoire d'un corpus massif de textes mémorisés en tant qu'ils exemplifient ces formes remarquables ».

47. La séquence est conçue comme "une structure ", c'est-à-dire d'une part, "un réseau relationnel décomposable en parties reliées entre elles et reliées au tout qu'elles constituent » et d'autre part, " une entité relativement autonome, dotée d'une organisation préformatée interne qui lui est propre et donc en relation de dépendance-indépendance avec l'ensemble plus vaste dont elle est une partie constituante : le texte », J.-M. Adam, Les textes: types et prototypes [3 ${ }^{\mathrm{e}}$ édition], Paris, Armand Colin, coll. «Cursus », 2011, p. 44.

48. Pour Bernard Bray, les passages de narration constituent des séquences détachables appelées à être partagées : «Ces fragments sont relativement faciles à isoler et se prêtent bien à la lecture à haute voix ", "Mme de Sévigné et l'art de la narration", art. cit., p. 296. L'hypothèse que certaines portions de texte constituent « un segment textuel refermé sur lui-même, et qui sera soumis [...] à une appréciation séparée » ou encore une « forme courte à l'intérieur d'une forme courte» (ibid., p. 294 et 297) a bien valeur programmatique, dans la mesure où elle peut être appliquée à d'autres séquences que les séquences narratives.

49. A. Chamayou, «Une forme contre les genres: penser la littérature à travers les lettres du XVIII ${ }^{\mathrm{e}}$ siècle ", dans B. Melançon (dir.), Penser par lettre. Actes du colloque d'Azay-le-Ferron (mai 1997), Montréal, Fides, 1998, p. 253. «Les lettres, en effet, portent fréquemment la trace d'[...]écritures secondes, écritures en quête d'adresse, styles ou modalités en cours de formation, éléments de genre recomposés, et qui trouvent dans la forme épistolaire une résolution transitoire. Tout épistolier est ainsi essentiellement polygraphe. L'absence de statut générique de la lettre ouvre à l'instant l'éventail de tous les autres genres possibles. Pour le lecteur se manifestent alors le travail complexe du texte, les libres mécanismes de l'écriture. La lecture se trouve ainsi orientée vers un déchiffrement des divers indices génériques qui, sans viser leur harmonisation, révèle sous un nouvel éclat la contribution de chacun d'eux à l'ordre esthétique du texte." (ibid., p. 252-253).

50. J. Jouvancy, Candidatus Rhetoricae, Cologne, S. Noethen, 1715, VII « De modo scribendae epistolae », 2 « Exempla epistolarum è Tullio sumpta », p. 404-413.

51. Telle est la définition que donne J.-M. Adam des cinq types de séquences de base qu'il retient (agencement narratif, argumentatif, explicatif, dialogal et descriptif), La linguistique textuelle [ $3^{\mathrm{e}}$ édition], Paris, Armand Colin, 2011, p. 162.

52. On se souvient de la satisfaction revendiquée par Roger Duchêne au seuil de la version publiée de sa thèse, dans une préface intitulée « Vingt-deux ans après » : «J'ai soutenu en 1969 la thèse qui me paraissait la plus propre à entrer par la bonne porte dans le monde sévignéen. Quoi qu'on ait écrit pour ou contre depuis, elle me paraît toujours la meilleure. Je maintiens donc. Et l'agacement ressenti en lisant sous mainte plume, qui oublie de me citer, que les lettres de Mme de Sévigné à sa fille sont des lettres d'amour est largement compensé par la satisfaction de voir que cette idée est désormais devenue une évidence [...]. » (Mme de Sévigné et la lettre d'amour, op. cit., p. VII).

53. Dans la lignée des propositions pionnières de sa Rhétorique de la lecture (op. cit.), Michel Charles effectue une micro-lecture des lettres de Mme de Sévigné sur le mariage avorté de Mademoiselle fondée sur l'analyse des dysfonctionnements du texte, Introduction à l'étude des textes, Paris, Seuil, coll. « Poétique », p. 249-259. 
54. Les séances du séminaire mensuel de la composante scientifique « $R$ ARE-Rhétorique de l'Antiquité à la Révolution ", dirigée à l'Université Grenoble Alpes par Ch. Noille, sont l'occasion de faire l'expérience des difficultés à mettre en forme un déroulé discursif qui ne coïncide jamais exactement avec les descriptifs théoriques des différents types de discours — d'où des discussions animées autour des focalisations inattendues, de la place flottante des arguments ou encore des interpolations imprévisibles. Si chaque texte soumis à l'exercice rhétorique de formalisation suscite des incertitudes quant à l'identification de ses modèles, c'est bien parce que «le type n'est pas une norme ", comme y insiste F. Goyet, "Le problème de la typologie des discours », Exercices de rhétorique [En ligne], 1 | 2013, URL : http://rhetorique.revues.org/122

55. Afin d'illustrer le fait que l'établissement d'une identification relativement opérationnelle n'interdit pas de proposer d'autres formalisations possibles, Christine Noille expérimente trois mises en forme concurrentes du même texte (un discours de Germanicus étant tour à tour décrit comme un discours de conciliatio, un discours d'objurgatio et un discours de menace), « Rhétorique de la lecture et compétence formalisatrice », en ligne dans l'Atelier de théorie littéraire du site Fabula sur www.fabula.org. Voir également la mise en perspective par Christine Noille de trois commentaires d'un discours d'Énée (celui de Vossius, celui de Lauban et celui de Ferrazzi), qui ni ne structurent le texte de la même façon ni n'actualisent les mêmes modèles, « Le discours d'un Prince (Énéide, I, 597-610). Archéologie de la disposition », Exercices de rhétorique [En ligne], 2 | 2013, URL : http : //rhetorique.revues.org/176

56. Glosant la distinction que fait Michel Charles entre une culture du «commentaire » et une culture «rhétorique " (L'Arbre et la Source, Paris, Seuil, 1985, p. 12), Christine Noille oppose deux conceptions du rapport au texte : « d'un côté, l'idée de l'autorité et de la monumentalité du texte détermine une enquête qui en déploie toujours plus les richesses et la singularité ; de l'autre, l'idée que le texte est le composé, la combinatoire - plus ou moins arbitraire, plus ou moins contingente - , de structures et de formes reproductibles et modifiables s'accorde avec une pratique manipulatrice et interventionniste, qui opère une sérialisation toujours possible du texte sur d'autres corpus exemplifiant des formes comparables, sur d'autres versions exemplifiant des formes modifiées», Chr. Noille, «La forme du texte: rhétorique et/ou interprétation », Fabula-LhT, n 14, "Pourquoi l'interprétation ?», février 2015, URL : http:// www.fabula.org/lht/14/noille.html

57. Pour M. Charles, les "critères rationnels" sur lesquels doit se faire "la construction de modèles de lecture » sont les suivants : " élégance ou économie des hypothèses, efficacité dans le choix des perspectives, rentabilité de telle ou telle prise sur le texte », Introduction à l'étude des textes, op. cit., p. 264.

58. Ch. Noille, «Les lettres de Sévigné sont-elles informes ? Éléments pour une rhétorique de la disposition », art. cit., p. 29.

59. Id.

60. Sur la notion de texte possible, on se reportera à l'entrée correspondante dans l'« Atelier de théorie littéraire » du site Fabula, www.fabula.org

61. On aura reconnu les postulats sur lesquels repose le protocole de lecture promu par Michel Charles, que résume ainsi Christine Noille: "contestation de l'autorité du texte, de sa monumentalité, de sa cohérence ; ouvertures du texte sur d'autres textes possibles ; pluralisation du texte en cohérences locales concurrentes ", "Sur un exemple de Michel Charles ou comment composer (avec) des textes ", Poétique, $\mathrm{n}^{\circ} 169,2012$, p. 98.

62. «L'interprétation commence où l'expérimentation formalisatrice cède le pas à la décision, où l'on n'en reste pas à l'étape technique du relevé méthodique et exhaustif de tous les embrayeurs possibles de séquençage, de cohésion, de transition dans la mise en forme du sémantisme; mais où on bascule dans le choix et la prévalence d'un système de modélisation sur un autre.", Christine Noille, «Rhétorique de la lecture et compétence formalisatrice ", art. cit. Pour une réflexion sur la répartition des tâches entre rhétorique et herméneutique, voir, du même auteur, 
"Le commentaire rhétorique classique, un modèle de micro-lecture non herméneutique ", dans Fabula-LHT n 3, www.fabula.org/lht/3/Noille.html

63. Ch. Noille, "Le discours d'un Prince (Énéide, I, 597-610). Archéologie de la disposition», art. cit., § 17.

64. M. Charles, Rhétorique de la lecture, op. cit., p. 118.

65. M. Charles, Rhétorique de la lecture, op. cit., p. 118.

\section{AUTEUR}

\section{CÉCILE LIGNEREUX}

Université Grenoble Alpes - UMR LITT\&ARTS / RARE Rhétorique de l'Antiquité à la Révolution 